\title{
Periodontal Disease Activity Measured by the Benzoyl-DL-Arginine- Naphthylamide Test Is Associated With Preterm Births
}

\author{
Hui-Chen Chan, ${ }^{*}$ Chen-Tsai Wu, ${ }^{\dagger}$ Kathleen B. Welch, ${ }^{\ddagger}$ and Walter J. Loesche ${ }^{\S}$
}

Background: Infection is a risk factor for preterm birth. This study was conducted in the field and addressed the link between periodontal pathogens measured with the benzoylDL-arginine-naphthylamide (BANA) test and preterm birth.

Methods: This prospective study was performed in Changhua, Taiwan. Periodontal examinations included the plaque index, papillary bleeding scores, and measurement of the BANA enzyme in plaque samples at the second and third trimesters. Independent variables included maternal demographic characteristics, previous pregnancy histories, risk factors, plaque and gingivitis scores, and current pregnancy outcomes.

Results: There were 19 (7\%) preterm deliveries among the 268 subjects. A history of a previous preterm birth and low birth weight, frequency of prenatal visits, preterm uterine contractions, antepartum hemorrhages, placenta previae, and preterm premature rupture of membranes were significantly related to preterm birth $(P=0.035,0.027,<0.001,0.025$, $0.006,0.014$, and $<0.001$, respectively). Maternal weight gain was higher with a normal term delivery $(P=0.003)$. Multivariable logistic regression analyses showed that the number of BANA-infected sites in the third trimester (odds ratio [OR]: 5.89; 95\% confidence interval [CI]: 1.5 to 31.6), maternal weight gain (OR: $0.78 ; 95 \% \mathrm{CI}: 0.65$ to 0.91 ), antepartum hemorrhages (OR: 10.0; 95\% CI: 2.2 to 46.9 ), and preterm premature rupture of membranes (OR: 12.6 ; $95 \% \mathrm{CI}: 3.97$ to 42.71) had significant influences on preterm-birth outcomes.

Conclusions: BANA-positive plaque in the third trimester was associated with preterm births after controlling for other risk factors. The BANA test can be used to screen pregnant women at chairside and/or bedside to apply suitable intervention tactics. J Periodontol 2010;81:982-991.

\section{KEY WORDS}

BANA; periodontal disease; preterm birth.

\footnotetext{
* Department of Orthodontic and Pediatric Dentistry, School of Dentistry, University of Michigan, Ann Arbor, MI.

† Dental Department, Show Chawn Memorial Hospital, Changhua, Taiwan.

$\neq$ Center for Statistical Consultation and Research, University of Michigan.

$\S$ Marcus Ward Professor Emeritus School of Dentistry, Professor Emeritus of Microbiology \& Immunology, School of Medicine, University of Michigan.
}

$\mathrm{T}$ The prevalence of preterm birth $(P B)$ is often associated with infections and/or inflammations ${ }^{1}$ because of the activation of the immune system, by microorganisms that trigger the release of inflammatory cytokines such as interleukin-8, interleukin-1, and tumor necrosis factor-alpha. ${ }^{1}$ This inflammatory cascade and microbial endotoxins derived from invading bacteria stimulate the production of prostaglandins, ${ }^{2}$ which can increase uterine contractions that result in preterm labor causing a PB. ${ }^{3}$ Among the infections that have been associated with PBs is periodontal disease. ${ }^{1}$

Periodontal disease is an inflammatory process in the periodontal tissue that is initiated by the microorganisms in the subgingival plaque. ${ }^{4}$ Although the microbiota of the subgingival plaque is complex, there appears to be a selection for anaerobes when there is an active disease process, especially for the Gram-negative, proteolytic species Porphyromonas gingivalis, Tannerella forsythia (previously $T$. forsythensis), and Treponema denticola. ${ }^{5}$ P. gingivalis, $T$. forsythia, and $T$. denticola were found in higher levels in subgingival plaques of women who delivered PB or low-birth weight (LBW) babies compared to women who delivered normal term (NT) babies. ${ }^{6,7}$ This association was concomitant with an increase in maternal 
serum inflammatory mediators and antibodies. ${ }^{6-8} P$. gingivalis was also detected in the amniotic cavity of pregnant women with threatened premature labor $^{7-9}$ and along with other periodontal species in the placentas of women with preeclampsia. ${ }^{10}$

Efforts to detect these periodontal pathogens in dental plaque have included DNA probes, cultural procedures, microscopic measures, and immunologic reagents. $P$. gingivalis, $T$. forsythia, and $T$. denticola possess a trypsin-like enzyme that can hydrolyze the synthetic trypsin substrate benzoylDL-arginine-naphthylamide (BANA). ${ }^{11}$ The presence of these organisms in subgingival plaque can be determined by the ability of the plaque to hydrolyze BANA $^{12}$ using a 5-minute chairside assay. In the detection of $P$. gingivalis, $T$. forsythia, and $T$. denticola, the BANA test had a $92 \%$ sensitivity and a $70 \%$ specificity compared to DNA probes and polyclonal immunologic reagents. ${ }^{13}$ When the BANA test was compared to checkerboard DNA-DNA hybridization using highly specific, whole-genomic DNA probes to $P$. gingivalis, $T$. forsythia, and $T$. denticola, it had a sensitivity of $95 \%$ and was most effective for the detection of these organisms when their levels in subgingival plaque were high, i.e., in the initial diagnosis of chronic periodontitis. ${ }^{14}$ The results suggest that the BANA test could be used as a surrogate for DNA probes in the detection of these bacterial species in plaque samples.

Probing measurement of pocket depth and attachment often require a separate visit to a dental clinic. The BANA test can be obtained at chairside and/or bedside ${ }^{15}$ and was shown to be significantly associated with probing depth ${ }^{16,17}$ and to predict future attachment loss after initial treatment. ${ }^{18}$ The BANA test was used in epidemiologic studies where it was found to be an important explanatory variable for attachment loss in seniors ${ }^{19}$ and to correlate with the Community Periodontal Index of Treatment Needs index. ${ }^{16}$ This suggested that the BANA test could be used under field conditions, such as the waiting room of a hospital, to obtain information concerning the periodontal status of the patient. The purpose of this prospective study was to investigate the relationship between the presence of BANA-positive species in subgingival plaque during the second and third trimesters with the subsequent development of PB under field conditions.

\section{MATERIALS AND METHODS}

\section{Participants}

This study was conducted in the Obstetric and Gynecological (OBGYN) clinics in Show Chawn Memorial Hospital and San Ann Hospital in Changhua, Taiwan, after all procedures were reviewed and approved by the Health Sciences Institutional Research Board of the University of Michigan (Hum00000327) and Hospital Institutional Research Board of the Show Chawn Memorial Hospital (940607). Subjects were recruited between September 2005 and June 2006 during their second-trimester visit to the OBGYN clinic.

\section{Experimental Protocol}

Pregnant women with a singleton gestation in the second trimester (gestational age: 13 to 28 weeks) were recruited, whereas subjects with multiple gestations who were undergoing fertility treatment were excluded. The subjects signed consent forms after the format, purpose, and nature of the study were presented to them. Information regarding maternal characteristics, which included demographic information data, previous pregnancy histories, and risk factors (history of smoking and alcohol consumption), was collected via a written questionnaire. At the secondtrimester appointment, dental measurements, including the plaque index score $(\mathrm{PI})^{20}$ and papillary bleeding score (PBS) ${ }^{21}$ were made, and measurement of the BANA enzyme in plaque samples using the BANA test ${ }^{\|}$was performed. The PI, PBS, and BANA test were repeated when the subjects returned for their third-trimester visit (gestational age: 25 to 40 weeks). Pregnancy outcomes, which were collected from hospital records, included the patient's status during pregnancy (gestational age at first prenatal visit, frequency of prenatal visits, prepregnancy weight and height, and weight and height at the last prenatal visit), complications during pregnancy, type of delivery, gender of the infant, gestational age, and birth weight at delivery. The participants did not have to make any visits other than their regularly scheduled prenatal visits.

\section{Dental Measurements}

Because periodontal disease frequently begins subgingivally in the interdental papilla around the posterior teeth, plaque samples from the four interdental sites between the first and second molars of each quadrant were collected. If teeth were missing, the plaque sample was removed from the mesial or distal side of the remaining tooth.

The PI score was recorded on a scale of 0 to 3 at the interdental site from which the plaque sample would be obtained for the BANA test. No plaque disclosing solution was used. After the PI was measured, the supragingival plaque was removed from the site, and a soft wooden toothpick " was inserted between the first and second molars in each quadrant. When the toothpick was removed, the PBS ${ }^{21}$ was recorded at the interdental papilla on a scale from 0 to 5. The subgingival plaque adherent to the toothpick was

|| BANAMet LLC, Ann Arbor, MI.

II STIM-U-DENT, Johnson \& Johnson, New Brunswick, NJ. 
used for the BANA test. A separate toothpick was used to obtain each plaque sample, and both sides of each toothpick were wiped onto the lower strip of the BANA card. The upper strip of the BANA card was moistened with distilled water, and the card was folded at the perforation mark so that the lower and upper reagent strips met. The folded card was incubated in a special BANA test-designed heater at $55^{\circ} \mathrm{C}$ for 5 minutes. After incubation, the lower reagent strip containing plaque was discarded in a manner appropriate for contaminated material. The color on the upper strip was recorded by the consensus of two examiners (HC and Natalie Grossman, BANAMet, Ann Arbor, MI) with no blue = negative, faint blue $=$ weakly positive, and blue $=$ positive. The intraexaminer $\kappa$ agreement was 0.92, and the interexaminer $\kappa$ agreement was 0.90 . For statistical analyses, weakly positive and positive results were recorded as positive. A woman was defined as being BANA infected when plaques from $\geq 2$ of the four sample sites were either weakly or strongly BANA positive.

\section{Definition of Pregnancy Outcomes}

The primary outcome was PB, which was defined as a birth occurring $<37$ weeks ( $<259$ days) of gestation and referred to the time that elapsed between the first day of the last menstrual period and the day of delivery. ${ }^{22}$ The obstetricians (Hong-Chen Chang, JinnFa Bai, and Biau Hsiung Chen, San Ann Hospital; and Hui-Yin Chiu, Show Chawn Memorial Hospital, Changhua, Taiwan) were masked to the subjects participating in the study and had no knowledge of the women's periodontal data.

\section{Statistical Analyses}

Power calculations were made prior to initiation of the study, assuming that the total PB rate in Taiwan is $\sim 7 \%^{23}$ and that $60 \%$ of our participants would be BANA positive. ${ }^{24}$ We expected to be able to recruit 300 patients in the time frame of the study and calculated that a two-group $\chi^{2}$ test with a 0.05 one-sided significance level would have $76 \%$ power to detect the difference between a $10 \%$ incidence of PB babies in the BANA-positive group and 3\% PB babies in the BANA-negative group, assuming sample sizes in the two groups of 180 and 120, respectively. Power calculations were performed using statistic software. ${ }^{\#}$ Independent variables included maternal demographic characteristics (age, occupational level, educational level, ethnicity, and previous medical history), pregnancy history (previous pregnancies, previous PBs, previous LBW infants, and abortion history), risk factors for PB (prepregnancy body mass index [BMI], smoking, and alcohol consumption), complications during pregnancy, second- and thirdtrimester dental measurements (the PI, PBS, and BANA test). Frequency data were generated for each categoric variable (e.g., educational level and ethnicity). Chi-square tests were calculated to assess the relationship of categoric variables (e.g., ethnicity) with PB (yes or no). Independent samples $t$ tests were carried out to determine whether there was a significant difference in the means of continuous variables (e.g., time of dental measurement) for PB versus NT outcomes. The potential independent variables for PB included in the multivariable logistic regression model were antepartum hemorrhage, a preterm premature rupture of the membrane, infected second and third BANA plaque samples, ethnicity, and weight gain during pregnancy. The statistical significance of each variable and odds ratio (OR) were calculated using the Firth bias correction ${ }^{25}$ for a small sample size. The various predictors were entered in different combinations into the models, and the model with the highest Nagelkerke maximum rescaled $R^{2}$ value ${ }^{26}$ and lowest Akaike's information criterion (AIC) ${ }^{27}$ value was selected. An $\alpha$ level of 0.05 was used for all statistical tests. Statistical analyses were carried out using a software package. **

\section{RESULTS}

There were 317 women who consented to participate in this study, of whom 13 were seen in the Show Chawn Memorial Hospital and 304 were seen in the San Ann Hospital. Eighteen subjects were excluded for the following reasons: multiple gestations $(n=1)$, early pregnancy termination $(n=2)$, and no information on the pregnancy outcome $(n=15)$. As a result, 299 subjects had BANA test results obtained in the second and/or third trimesters. To address the effect of BANA-infected plaque on pregnancy outcomes, the 268 subjects who had BANA test results in the second and third trimesters and pregnancy outcomes were included in the statistical analysis. Of these 268 subjects, 194 (72.4\%) subjects had $>10$ prenatal appointments. A total of 207 women (77.2\%) delivered their babies vaginally, and 61 subjects $(22.8 \%)$ underwent cesarean section deliveries. Nineteen women had a PB outcome (7\%). There was no significant difference in gender between PB and NT infants $(P>0.999)$.

\section{Maternal Demographic Characteristics}

The ages of subjects ranged from 16 to 43 years with a mean \pm SD of $27.2 \pm 4.3$ years. Sixty-seven women were $\geq 30$ years of age. There was no significant difference in maternal age, occupational level, educational level, and previous medical history among subjects who had PBs and subjects who had NT births (Table $1)$. Thirteen of 268 subjects were non-Taiwanese,

\footnotetext{
\# nQuery Advisor version 7.0, Statistical Solutions, Cork, Ireland. * PASW Statistics 18, SPSS Inc, Chicago, IL; SAS for Windows, release 9.2, 2002-2008, SAS Institute, Cary, NC.
} 
Table I.

\section{Relationship of Maternal Characteristics to PB for 268 Subjects}

\begin{tabular}{|c|c|c|c|}
\hline \multirow[b]{2}{*}{ Variables } & \multicolumn{2}{|c|}{ Pregnancy Outcome } & \multirow[b]{2}{*}{$p^{*}$} \\
\hline & Normal $(n=249)(n[\%])$ & $\operatorname{PB}(n=19)(n[\%])$ & \\
\hline \multicolumn{4}{|l|}{ Demographic characteristic } \\
\hline \multicolumn{4}{|l|}{ Age (years) } \\
\hline$<30$ & $188(75.5)$ & $13(68.4)$ & 0.582 \\
\hline$\geq 30$ & $61(24.5)$ & $6(31.6)$ & \\
\hline \multicolumn{4}{|l|}{ Ethnicity } \\
\hline Taiwanese & $239(96.0)$ & $16(84.2)$ & 0.055 \\
\hline Non-Taiwanese & $10(4.0)$ & $3(15.8)$ & \\
\hline \multicolumn{4}{|l|}{ Occupation } \\
\hline Housewife & $88(35.3)$ & $5(26.3)$ & 0.618 \\
\hline Non-housewife & $161(64.7)$ & 14 (73.7) & \\
\hline \multicolumn{4}{|l|}{ Education } \\
\hline Less than senior high school & $132(53.0)$ & $12(63.2)$ & 0.478 \\
\hline Senior high school or further & $117(47.0)$ & $7(36.8)$ & \\
\hline \multicolumn{4}{|l|}{ Previous medical history } \\
\hline Healthy & $228(91.6)$ & 18 (94.7) & $>0.999$ \\
\hline Medical condition & $21(8.4)$ & I (5.3) & \\
\hline \multicolumn{4}{|l|}{ Previous pregnancy history } \\
\hline \multicolumn{4}{|l|}{ First-time pregnancy } \\
\hline Yes & III (44.6) & $8(42.1)$ & $>0.999$ \\
\hline No & $138(55.4)$ & I| (57.9) & \\
\hline \multicolumn{4}{|l|}{ Number of previous pregnancies } \\
\hline 0 & ||| (44.6) & $8(42.1)$ & 0.753 \\
\hline I to 2 & $121(48.6)$ & $9(47.4)$ & \\
\hline$\geq 3$ & $17(6.8)$ & $2(10.5)$ & \\
\hline \multicolumn{4}{|l|}{ Previous PB } \\
\hline Yes & $8(3.2)$ & $3(15.8)$ & 0.035 \\
\hline No & $24 \mid(96.8)$ & $16(84.2)$ & \\
\hline \multicolumn{4}{|l|}{ Previous LBW } \\
\hline Yes & $7(2.8)$ & $3(15.8)$ & 0.027 \\
\hline No & $242(97.2)$ & $16(84.2)$ & \\
\hline \multicolumn{4}{|l|}{ Previous spontaneous abortion } \\
\hline Yes & $30(12.0)$ & $0(0.0)$ & 0.143 \\
\hline No & $219(88.0)$ & $19(100.0)$ & \\
\hline \multicolumn{4}{|l|}{ Previous artificial abortion } \\
\hline Yes & $42(16.9)$ & $3(15.8)$ & $>0.999$ \\
\hline No & $207(83.1)$ & $16(84.2)$ & \\
\hline \multicolumn{4}{|l|}{ Risk factor } \\
\hline \multicolumn{4}{|l|}{ Prepregnancy BMI $\left(\mathrm{kg} / \mathrm{m}^{2}\right)$} \\
\hline$<19.8$ & $102(41.0)$ & $9(47.4)$ & 0.633 \\
\hline$\geq 19.8$ & $147(59.0)$ & $10(52.6)$ & \\
\hline \multicolumn{4}{|l|}{ Smoking } \\
\hline Never & $245(98.4)$ & $19(100.0)$ & $>0.999$ \\
\hline Now & $4(1.6)$ & $0(0.0)$ & \\
\hline \multicolumn{4}{|l|}{ Alcohol drinking } \\
\hline Never & $247(99.2)$ & $19(100.0)$ & $>0.999$ \\
\hline Now & $2(0.8)$ & $0(0.0)$ & \\
\hline \multicolumn{4}{|l|}{ First prenatal care } \\
\hline$<12$ weeks & $244(98.0)$ & $17(89.5)$ & 0.127 \\
\hline 13 to 20 weeks & $4(1.6)$ & $2(10.5)$ & \\
\hline$>20$ weeks & I (0.4) & $0(0.0)$ & \\
\hline
\end{tabular}


Table I. (continued)

Relationship of Maternal Characteristics to PB for 268 Subjects

\begin{tabular}{lcrc}
\hline & \multicolumn{2}{c}{ Pregnancy Outcome } & \multirow{2}{*}{$P^{*}$} \\
\cline { 2 - 3 } Variables & Normal $(n=249)(n[\%])$ & $P B(n=19)(n[\%])$ & $<0.001$ \\
\hline Prenatal visit & $57(22.9)$ & $17(89.5)$ & \\
$\leq 10$ visits & $192(77.1)$ & $2(10.5)$ & \\
$>10$ visits & &
\end{tabular}

* Fisher exact test.

and these women had a higher proportion of PBs than the Taiwanese women $(P=0.055)$. None of these non-Taiwanese women had graduated from senior high school, whereas $\sim 50 \%$ of the Taiwanese women had schooling beyond high school.

\section{Previous Pregnancy History (Table 1)}

A total of 119 women (44.4\%) were pregnant for the first time, and they were no more likely than the multiparious women to have a PB (>0.999; Table 1). Pregnant women who had a previous PB and previous LBW infants were more likely to have a PB $(P=0.035$ and 0.027, respectively).

\section{Risk Factors and Pregnancy Complications}

There was no significant relationship between a PB and prepregnancy BMI, smoking, alcohol consumption during pregnancy, and time of the first prenatal visit $(P>0.05$ for all comparisons; Table 1$)$. The BMI at the first prenatal visit for the women destined to be in the NT group (mean: 21.04; SD: 3.57) and women destined to be in the PB group (mean: 20.61; SD: 2.33) was comparable $(P=0.604)$. Thereafter, women in the NT group gained significantly more weight than the women in the PB group $(P=$ 0.003 ) (data not shown). Ninety-eight percent of the women never smoked and the four women who were current smokers were in the NT group. Only two women reported consuming alcoholic beverages (Table 1). There was a highly significant increase in PB for women who had a preterm uterine contraction, antepartum hemorrhage, placenta previa, and preterm premature rupture of membrane $(P=0.025$, $0.006,0.014$, and $<0.001$, respectively) (Table 2 ).

\section{Dental Measurements}

Approximately $80 \%$ of the women had good or fair oral hygiene, and there was no change in oral hygiene as they progressed from the second to third trimesters (Table 3). Sixty-six percent of the women had gingivitis in the second trimester, and this prevalence increased significantly to $78 \%$ in the third trimester. Women who were BANA positive (i.e., $\geq 2$ sites of the four sampling sites were positive or weakly positive) were more likely to have gingival bleeding in the second and third trimesters $(P<0.001$ and $P<0.001$, respectively) than women who were BANA negative (fewer than two of the four sampling sites) (data not shown). There was a tendency for the prevalence of a BANA infection to increase from the second to third trimesters (Table 3).

There were no significant differences in the gestational age at the time of the examination among the women destined to have an NT birth or a PB (Table $4)$. In the second and third trimesters, there was no significant difference among women who had PBs and women who had NT births in the adequacy of oral hygiene procedures or in the presence of gingivitis, although there was a slight tendency for the prevalence of gingivitis to be higher in the second trimester in women destined to have a PB (Table 4). In the third trimester, but not the second trimester, there was a tendency for women with $\geq 2$ BANA-positive sites to be in the PB group compared to women with $<2$ BANA-positive sites $(P=0.08)$ (Table 4). Although the BANA results for each individual did not change between the second and third trimesters for the majority of the subjects, there was a tendency for a BANA infection to decrease in the NT subjects compared to the PB subjects in the third trimester. Twelve percent of the NT subjects had a decrease in BANA infections, whereas only $5 \%$ of PB subjects had a decrease. Twenty-six percent of the PB subjects showed an increase in BANA infections, whereas $17 \%$ of the NT subjects showed an increase in BANA infections (data not shown). The sample size was too small to show significance.

Logistic regression analysis was used to model the occurrence of PBs based on the values of the various explanatory variables. The following variables were not significant in any of the models: age, education and occupation, smoking status, alcohol consumption, oral hygiene status, presence of gingivitis, secondtrimester BANA results, prepregnancy BMI, number of previous pregnancies, and whether the pregnancy was the subject's first pregnancy. The model with the highest maximum square value and lowest AIC is shown in Table 5. BANA-infected plaque samples in the third trimester (OR: 5.9), ethnicity (OR: 5.6), 
Table 2.

\section{Relationship of Pregnancy Complications to PB for 268 Subjects}

\begin{tabular}{|c|c|c|c|}
\hline Variable & Normal $(n=249)(n[\%])$ & PB $(n=19)(n[\%])$ & $p^{*}$ \\
\hline \multicolumn{4}{|c|}{ Genitourinary infection } \\
\hline No & $182(73.1)$ & $13(68.4)$ & \\
\hline Yes & $67(26.9)$ & $6(31.6)$ & 0.605 \\
\hline \multicolumn{4}{|c|}{ Upper-respiratory infection } \\
\hline No & $|4|(56.6)$ & $7(36.8)$ & \\
\hline Yes & $108(43.4)$ & $12(63.2)$ & 0.149 \\
\hline \multicolumn{4}{|c|}{ Hemorrhage at $<28$ weeks } \\
\hline No & $193(77.5)$ & $17(89.5)$ & \\
\hline Yes & $56(22.5)$ & $2(10.5)$ & 0.384 \\
\hline \multicolumn{4}{|c|}{ Preterm uterine contraction } \\
\hline No & $220(88.4)$ & $13(68.4)$ & \\
\hline Yes & $29(11.6)$ & $6(31.6)$ & 0.025 \\
\hline \multicolumn{4}{|c|}{ Antepartum hemorrhage } \\
\hline No & $24 \mid(96.8)$ & $15(78.9)$ & \\
\hline Yes & $8(3.2)$ & $4(21.1)$ & 0.006 \\
\hline \multicolumn{4}{|c|}{ Placenta previa } \\
\hline No & $248(99.6)$ & $17(89.5)$ & \\
\hline Yes & I (0.4) & $2(10.5)$ & 0.014 \\
\hline \multicolumn{4}{|l|}{ PPROM } \\
\hline No & $236(94.8)$ & II (57.9) & \\
\hline Yes & $13(5.2)$ & $8(42.1)$ & $<0.001$ \\
\hline \multicolumn{4}{|l|}{$\mathrm{PIH}$} \\
\hline No & $244(98.0)$ & $19(100.0)$ & \\
\hline Yes & $5(2.0)$ & $0(0.0)$ & $>0.999$ \\
\hline \multicolumn{4}{|l|}{ PGD } \\
\hline No & $247(99.2)$ & $19(100.0)$ & \\
\hline Yes & $2(0.8)$ & $0(0.0)$ & $>0.999$ \\
\hline
\end{tabular}

PPROM = Preterm premature rupture of membrane; PIH = pregnancy-induced hypertension; PGD = pregnancy gestational diabetes .

* Fisher exact test.

antepartum hemorrhage (OR: 10.0), and the preterm premature rupture of membranes (OR: 12.6) were significantly positive predictors of PBs, whereas maternal weight gain (OR: 0.78) was a significantly negative predictor of PBs, after adjusting for other potential risk factors. Although BANA-infected plaque samples in the second trimester were not significantly related to PBs, this variable was kept in the model because of its contrast with the third-trimester BANA results.

\section{DISCUSSION}

Periodontal disease is traditionally defined on the basis of clinical morbidity about the teeth, such as probing depth, clinical attachment loss, and radiographic bone loss. There is no consensus as to what constitutes the threshold for periodontal disease as documented by the fact that at least 14 different definitions and 50 different measurements have been used to associate periodontal disease with PB and/or LBW infants. ${ }^{28}$ This variability in disease definition has lead to contradictory results with regard to the role of periodontal disease in adverse pregnancy outcomes. ${ }^{28}$ Although periodontal disease is regarded as an infection, only occasionally are markers of infection used to recognize this infection, and then it is usually the host response, as noted by bleeding, the visual appearance of tissue inflammation, and the presence of inflammatory markers. This current prospective study analyzed periodontal pathogens in dental plaque, albeit indirectly by an enzyme test, to address the association between periodontal disease and adverse pregnancy outcomes. Our population-based study demonstrated a link between BANA-positive dental plaques in the third trimester and PBs.

Periodontal disease, as a source of persistent infection, has been indicated by increased serum $\mathrm{C}$-reactive 
protein (CRP) levels. ${ }^{29}$ In pregnant women, elevated CRP levels were associated with periodontal disease in African American women ${ }^{30}$ and with an increased risk of preeclampsia. ${ }^{31}$ In this regard, the value of microbial tests to diagnose a periodontal infection would seem worthwhile. Although the subgingival plaque flora is bacteriologically complex, $P$. gingivalis, $T$. forsythia, and $T$. denticola have emerged as major periodontal pathogens. ${ }^{5}$ These species were associated

Table 3.

\section{Comparison of Categoric Variables for Dental Measurements Between the Second and Third Trimesters}

\begin{tabular}{|c|c|c|c|}
\hline Variables & $\begin{array}{l}\text { Second Trimester } \\
\qquad(n=268) \\
(n[\%])\end{array}$ & $\begin{array}{l}\text { Third Trimester } \\
\qquad(n=268) \\
(n[\%])\end{array}$ & p* \\
\hline $\begin{array}{l}\text { Oral hygiene } \\
\text { Good or fairt } \\
\text { Poor }\end{array}$ & $\begin{array}{r}214(79.9) \\
54(20.1)\end{array}$ & $\begin{array}{r}223(83.2) \\
45(16.8)\end{array}$ & 0.306 \\
\hline $\begin{array}{l}\text { Gingivitis } \\
\text { No } \\
\text { Yes }^{\ddagger}\end{array}$ & $\begin{array}{r}92(34.3) \\
176(65.7)\end{array}$ & $\begin{array}{r}59(22) \\
209(78)\end{array}$ & $<0.001$ \\
\hline $\begin{array}{l}\text { BANA-infected } \\
\text { plaque samples } \\
\text { No } \\
\text { Yes }^{\S}\end{array}$ & $\begin{array}{l}112(4 \mid .8) \\
156(58.2)\end{array}$ & $\begin{array}{r}95(35.4) \\
173(64.6)\end{array}$ & 0.068 \\
\hline
\end{tabular}

* McNemar test.

$\dagger$ PI score $\geq 2$ in $<50 \%$ of sites.

\# Two or more sites bled after measurement by the toothpicks.

$\S$ Two or more sites that were BANA positive or weakly positive. with an increased risk for preterm delivery ${ }^{7}$ and were detected at higher levels in women who delivered preterm LBW infants ${ }^{6}$ and in the placentas of women with preeclampsis. ${ }^{10}$

DNA probes were used to establish the connection among $P$. gingivalis, $T$. denticola, and $T$. forsythia with adverse pregnancy outcomes in the cited studies. ${ }^{7,32}$ They were also used to show that periodontal treatment in the second trimester could significantly reduce the levels of these species in plaque samples. ${ }^{32}$ The use of DNA probes is a laboratory-based procedure that requires equipment and resources that were not available for the present study, whereas the BANA test could be performed at chairside and lended itself to the type of field study described. The BANA test appears to be a reliable surrogate for the use of DNA probes in the detection of $P$. gingivalis, $T$. denticola, and $T$. forsythia in plaque samples. ${ }^{14}$ Bayingana ${ }^{33}$ showed that the BANA test was more reflective of gingival conditions during pregnancy than were DNA probes.

Periodontal disease, as a response to a chronic infection, shares risk factors with a PB. ${ }^{1}$ The logistic regression model with the Firth correction for a small sample size was performed to control for the possible confounding effects of other predictors. This model indicated that the odds of having a PB were 5.9 times higher for women in the third trimester with $\geq 2$ infected BANA-positive or weakly BANA-positive sites compared to women with fewer than two BANA-positive or weakly BANA-positive sites after controlling for other variables. Other investigators ${ }^{6,7}$ obtained bacteriologic data from women in the second trimester and

Table 4.

\section{Relationship of Dental Measurements to PB}

\begin{tabular}{|c|c|c|c|c|c|c|}
\hline & \multicolumn{3}{|c|}{ Second Trimester $(n=268)$} & \multicolumn{3}{|c|}{ Third Trimester $(n=268)$} \\
\hline & Normal $(n=249)$ & $P B(n=19)$ & $P$ & Normal $(n=249)$ & $P B(n=19)$ & $P$ \\
\hline Gestational age at sampling ( $\mathrm{n}$ [mean days])* & $249(137)$ & $19(144)$ & 0.289 & 249 (209) & $19(2 \mid 2)$ & 0.619 \\
\hline $\begin{array}{l}\text { Oral hygiene }(n[\%])^{\dagger} \\
\text { Good or fairł } \\
\text { Poor }\end{array}$ & $\begin{array}{r}201(80.7) \\
48(19.3)\end{array}$ & $\begin{array}{r}13(68.4) \\
6(31.6)\end{array}$ & 0.233 & $\begin{array}{r}207(83.1) \\
42(16.9)\end{array}$ & $\begin{array}{r}16(84.2) \\
3(15.8)\end{array}$ & $>0.999$ \\
\hline $\begin{array}{l}\text { Gingivitis }(\mathrm{n}[\%])^{\dagger} \\
\text { No } \\
\text { Yes }\end{array}$ & $\begin{array}{r}88(35.3) \\
|6|(64.7)\end{array}$ & $\begin{array}{r}4(21.1) \\
15(78.9)\end{array}$ & 0.316 & $\begin{array}{r}55(22.1) \\
194(77.9)\end{array}$ & $\begin{array}{r}4(21.1) \\
15(78.9)\end{array}$ & $>0.999$ \\
\hline $\begin{array}{l}\text { BANA-infected plaque samples }(\mathrm{n}[\%])^{\dagger} \\
\text { No } \\
\text { Yes }\end{array}$ & $\begin{array}{l}105(42.2) \\
144(57.8)\end{array}$ & $\begin{array}{r}7(36.8) \\
12(63.2)\end{array}$ & 0.810 & $\begin{array}{r}92(36.9) \\
157(63.1)\end{array}$ & $\begin{array}{r}3(15.8) \\
16(84.2)\end{array}$ & 0.081 \\
\hline
\end{tabular}

* Independent samples $t$ test.

$\dagger$ Fisher exact test.

$\neq$ PI score $\geq 2$ in $<50 \%$ of sites.

$\S$ Two or more sites bled after measurement by the toothpicks.

\| Two or more sites were BANA positive or weakly positive. 
Table 5.

\section{Relationships of Predictors to PB Based on Logistic Regression Analysis for 268 Subjects Who Had Plaque Sampled for the BANA Test in the Second and Third Trimesters*}

\begin{tabular}{|c|c|c|c|c|c|}
\hline Explanatory Variables & df & P & OR & \multicolumn{2}{|c|}{$95 \% \mathrm{Cl}$ for $\mathrm{OR}$} \\
\hline Weight gain & । & 0.003 & 0.78 & 0.65 & 0.91 \\
\hline Antepartum hemorrhage & । & 0.004 & 10.00 & 2.16 & 46.90 \\
\hline Second-trimester BANA infection $§$ & । & 0.59 & 0.73 & 0.23 & 2.36 \\
\hline Third-trimester BANA infection $\S$ & । & 0.02 & 5.89 & 1.48 & 31.56 \\
\hline
\end{tabular}

$\mathrm{df}=$ degrees of freedom; $\mathrm{CI}=$ confidence interval.

* All variables were included in the model simultaneously. Age, gender, oral hygiene, gingivitis, history of PBs, history of LBW, and premature contraction were not significant in the model. Firth bias correction was used for the analysis Nagelkerke $\mathrm{R}^{2}=0.412 ; \mathrm{AIC}=94.87$.

$\S$ BANA-infection mean plaque removed from $\geq 2$ sites were BANA positive or weakly positive.

found that the levels of eight plaque bacteria, including $P$. gingivalis, $T$. forsy thia, and $T$. denticola, tended to be higher in the second trimester in mothers who delivered preterm babies than in mothers who delivered term babies. ${ }^{7}$ Our results show that $58 \%$ of the women in the second trimester had a BANA infection, but that no connection between a BANA infection and PB could be shown until the third trimester. This could have important implications for the timing of treatment.

Because periodontal disease is preventable and treatable, treating periodontal disease during pregnancy should improve pregnancy outcomes. Two large, well controlled, intervention studies ${ }^{34,35}$ that used debridement procedures (i.e., scaling and root planing was delivered in the second trimester) were unsuccessful in reducing PBs. A study ${ }^{36}$ that began in the second trimester and continued into the third trimester and included an antimicrobial agent (i.e., a $0.12 \%$ chlorhexidine rinse) in addition to debridement was successful in reducing PBs. This difference in outcomes suggests that scaling and root planing in the second trimester was not enough to reduce PBs. The importance of timing of the treatment was shown by a study ${ }^{37}$ in which women who had periodontal disease and were hospitalized with a threatening PB were randomly assigned to a treatment group or to a non-treatment group. ${ }^{37}$ The treatment was provided in the third trimester, at $\sim 32$ weeks, and consisted of oral hygiene instructions, scaling and root planning, and polishing of teeth with a fluoride paste. The babies of the treated women were delivered at 37.5 weeks and weighed $3,079 \mathrm{~g}$, which was significantly more than the 2,602-g infants who were born at 36.1 weeks to the women in the comparable control group.
In one intervention study, ${ }^{35}$ an average of 1.3 sessions of scaling and root planing was associated with a $\geq 2$-mm loss of attachment at $\geq 4$ sites in $41 \%$ of the women. Scaling and root planing often causes bacteremia, the intensity of which increases with the severity of periodontal disease, ${ }^{38-40}$ and increases the level of interleukin-6, ${ }^{41,42}$ which has been indicated as a risk for PB. ${ }^{43}$ It is possible that mechanical debridement without a concurrent usage of an antimicrobial agent may cause a bacteremia or incite an acute inflammatory response. ${ }^{44}$ In this regard, the strategy for reducing PBs by periodontal intervention might consider restricting the treatments to women who have a periodontal infection in the early third trimester, include the use of antimicrobial agents, and provide intervention at the gestational age of 28 to 32 weeks.

There is evidence that suggests that ethnicity might play a role in PBs. ${ }^{1}$ In our study, $4.9 \%$ of the subjects were non-Taiwanese and they were more likely to have a higher rate of an adverse pregnancy outcome compared to the Taiwanese women $(P=0.055)$. The non-Taiwanese women tended to have a lower social economic status as indicated by the educational level compared to the Taiwanese women (Table 1). Also, married immigrants faced problems of adaptation, communication difficulties, a lack of family support from their home town, and barriers to healthcare system use at the beginning of their lives in Taiwan. ${ }^{45}$

Smoking exhibits a dose-dependent relationship with PBs, as does a very high consumption of alcohol. ${ }^{1,46}$ In Taiwan, women rarely smoke or use alcohol or drugs, and especially do not do so during pregnancy. The self-reported data regarding smoking and alcohol consumption were only $1.5 \%$ and $0.7 \%$, 
respectively. As a result, our study provided information on the association between periodontal disease and PBs without these confounding factors. In Taiwan, the National Health Insurance provides for 10 paid prenatal examinations during pregnancy. Almost all (i.e., 98\%) of our participants had their first prenatal consultation at $<12$ weeks gestational age, which would eliminate inadequate prenatal care as a risk factor for a PB. ${ }^{47,48}$

A history of a PB or a history of LBW was not significant in the adjusted models. Both histories are usually risk factors for PBs. ${ }^{1}$ Possible reasons for the lack of significance include the small number of PBs or that the association of these factors with PBs is through an underlying BANA infection so that adjusting for BANA infection removes the association. We tested for the latter possibility by removing the BANA data from the models and still found no association of a history of a PB or LBW to be significant predictors.

In our population, women with $\leq 10$ prenatal visits had more PBs compared to women who had $>10$ prenatal visits $(P<0.001)$. This result is consistent with the shorter gestational age and lower weight gain observed in the PB women.

\section{CONCLUSIONS}

Traditional measurements for diagnosing periodontal disease focused on clinical morbidity and often resulted in an inconsistent diagnosis and the inability to recognize active disease. ${ }^{28}$ In this regard, studying the anaerobic bacterial burden and the inflammatory response may be more critical than measuring probing depths. To our knowledge, our study provides a new insight by addressing the infectivity progression with BANAassociated periodontal pathogens and suggests that the third-trimester bacterial status of the subgingival plaque may be an important predictor of PBs. The ability of the BANA test to detect anaerobic periodontal pathogens makes it a useful tool for chairside screening of at-risk populations such as pregnant women.

\section{ACKNOWLEDGMENTS}

This research was performed by Dr. Hui-Chen Chan in partial fulfillment of the requirements for a master's thesis in Pediatric Dentistry. The authors thank Dr. Hui-Yin Chiu at Show Chawn Memorial Hospital and Dr. Jinn-Fa Bai, Dr. Hong-Chen Chang and Dr. Biau Hsiung Chen, San Ann Hospital, for providing access to clinic patients and clinical facilities. Also, thanks to Mrs. Natalie Grossman, consultant for BANAMet, for her participation in helping to read BANA results, and to all of the subjects for participating in this research. The authors gratefully acknowledge Dr. Fenno, Department of Biologic \& Materials Sciences, School of Dentistry, University of Michigan, and Dr. Taichman, Department of Periodontics and Oral Medicine,
School of Dentistry, University of Michigan, for advice and guidance, and to BANAMet, Ann Arbor, Michigan, for their BANA tests and incubators. This research was funded by a gift from Oralife, Toronto, Ontario, to the School of Dentistry, University of Michigan. Dr. Loesche has received financial support from BANAMet and is a partner of BANAMet, the manufacturer of the BANA test. Drs. Chan, Wu, and Welch report no conflicts of interest related to this study.

\section{REFERENCES}

1. Goldenberg RL, Culhane JF, Iams JD, Romero R. Epidemiology and causes of preterm birth. Lancet 2008;371:75-84.

2. Romero R, Hobbins JC, Mitchell MD. Endotoxin stimulates prostaglandin E2 production by human amnion. Obstet Gynecol 1988;71:227-228.

3. Goldenberg RL, Hauth JC, Andrews WW. Intrauterine infection and preterm delivery. N Engl J Med 2000; 342:1500-1507.

4. Pihlstrom BL, Michalowicz BS, Johnson NW. Periodontal diseases. Lancet 2005;366:1809-1820.

5. Loesche WJ, Grossman NS. Periodontal disease as a specific, albeit chronic, infection: diagnosis and treatment. Clin Microbiol Rev 2001;14:727-752.

6. Offenbacher S, Jared HL, O'Reilly PG, et al. Potential pathogenic mechanisms of periodontitis associated pregnancy complications. Ann Periodontol 1998;3: 233-250.

7. Lin D, Moss K, Beck JD, Hefti A, Offenbacher S. Persistently high levels of periodontal pathogens associated with preterm pregnancy outcome. J Periodontol 2007;78:833-841.

8. Hasegawa K, Furuichi Y, Shimotsu A, et al. Associations between systemic status, periodontal status, serum cytokine levels, and delivery outcomes in pregnant women with a diagnosis of threatened premature labor. J Periodontol 2003;74:1764-1770.

9. León R, Silva N, Ovalle A, et al. Detection of Porphyromonas gingivalis in the amniotic fluid in pregnant women with a diagnosis of threatened premature labor. J Periodontol 2007;78:1249-1255.

10. Barak S, Oettinger-Barak O, Machtei EE, Sprecher H, Ohel G. Evidence of periopathogenic microorganisms in placentas of women with preeclampsia. J Periodontol 2007;78:670-676.

11. Loesche WJ. The identification of bacteria associated with periodontal disease and dental caries by enzymatic methods. Oral Microbiol Immunol 1986; 1:65-72.

12. Loesche WJ, Syed SA, Stoll J. Trypsin-like activity in subgingival plaque. A diagnostic marker for spirochetes and periodontal disease? J Periodontol 1987; 58:266-273

13. Loesche WJ, Lopatin DE, Giordano J, Alcoforado G, Hujoel P. Comparison of the benzoyl-DL-argininenaphthylamide (BANA) test, DNA probes, and immunological reagents for ability to detect anaerobic periodontal infections due to Porphyromonas gingivalis, Treponema denticola, and Bacteroides forsythus. J Clin Microbiol 1992;30:427-433.

14. de Andrade JA, Feres M, de Figueiredo LC, Salvador SL, Cortelli SC. The ability of the BANA Test to detect different levels of $P$. gingivalis, $T$. denticola and $T$. forsythia. Brazilian Oral Research 2010; in press. 
15. Rubenfire M, Grossman NS, Kaciroti N, Apsey DJ, Loesche WJ. Anaerobic dental flora and the acute coronary syndrome. Coron Artery Dis 2007;18:111116.

16. Grisi MF, Novaes AB, Ito IY, Salvador SL. Relationship between clinical probing depth and reactivity to the BANA test of samples of subgingival microbiota from patients with periodontitis. Braz Dent J 1998;9:77-84.

17. Apsey DJ, Kaciroti N, Loesche WJ. The diagnosis of periodontal disease in private practice. J Periodontol 2006;77:1572-1581.

18. Loesche WJ, Giordano J, Hujoel PP. The utility of the BANA test for monitoring anaerobic infections due to spirochetes (Treponema denticola) in periodontal disease. J Dent Res 1990;69:1696-1702.

19. Elter JR, Beck JD, Slade GD, Offenbacher S. Etiologic models for incident periodontal attachment loss in older adults. J Clin Periodontol 1999;26:113-123.

20. Silness, J. and H. Löe. Periodontal disease in pregnancy. II. Correlation between oral hygiene and periodontal condition. Acta Odontol Scand. 1964; 22:121-135.

21. Loesche WJ. Clinical and microbiological aspects of chemotherapeutic agents used according to the specific plaque hypothesis. J Dent Res 1979;58:24042412.

22. Berg AT. Menstrual cycle length and the calculation of gestational age. Am J Epidemiol 1991;133:585-589.

23. Ko YL, Wu YC, Chang PC. Physical and social predictors for pre-term births and low birth weight infants in Taiwan. J Nurs Res 2002;10:83-89.

24. Lee Y, Tchaou WS, Welch KB, Loesche WJ. The transmission of BANA-positive periodontal bacterial species from caregivers to children. J Am Dent Assoc 2006;137:1539-1546.

25. Firth, D. Bias Reduction of Maximum Likelihood Estimates. Biometrika 1993;80:27-38.

26. Nagelkerke, N. J. D. A note on a general definition of the coefficient of determination. Biometrika 1991;78: 691-692.

27. Akaike, H. "A new look at the statistical model identification". IEEE Transactions on Automatic Control 1974;19:716-723.

28. Manau C, Echeverria A, Agueda A, Guerrero A, Echeverria JJ. Periodontal disease definition may determine the association between periodontitis and pregnancy outcomes. J Clin Periodontol 2008;35:385397.

29. Slade GD, Offenbacher S, Beck JD, Heiss G, Pankow JS. Acute-phase inflammatory response to periodontal disease in the US population. J Dent Res 2000;79: 49-57.

30. Horton AL, Boggess KA, Moss KL, Jared HL, Beck J, Offenbacher S. Periodontal disease early in pregnancy is associated with maternal systemic inflammation among African American women. J Periodontol 2008; 79:1127-1132.

31. Ruma M, Boggess K, Moss K, et al. Maternal periodontal disease, systemic inflammation, and risk for preeclampsia. Am J Obstet Gynecol 2008;198:389. e1-389.e5.

32. Novak MJ, Novak KF, Hodges JS, et al. Periodontal bacterial profiles in pregnant women: response to treatment and associations with birth outcomes in the obstetrics and periodontal therapy (OPT) study. J Periodontol 2008;79:1870-1879.
33. Bayingana $\mathrm{C}$. The prevelance of members of the "red complex" in pregnant women as revealed by PCR and BANA hydrolysis. [Thesis]. Bellville, South Africa: University of the Western Cape; 2005;1-93.

34. Michalowicz BS, Hodges JS, DiAngelis AJ, et al. Treatment of periodontal disease and the risk of preterm birth. N Engl J Med 2006;355:1885-1894.

35. Offenbacher S, Beck JD, Jared HL, et al. Effects of periodontal therapy on rate of preterm delivery: a randomized controlled trial. Obstet Gynecol 2009; 114:551-559.

36. Lopez NJ, Da Silva I, Ipinza J, Gutierrez J. Periodontal therapy reduces the rate of preterm low birth weight in women with pregnancy-associated gingivitis. J Periodontol 2005;76:2144-2153.

37. Radnai M, Pal A, Novak T, Urban E, Eller J, Gorzo I. Benefits of periodontal therapy when preterm birth threatens. J Dent Res 2009;88:280-284.

38. Forner L, Larsen T, Kilian M, Holmstrup P. Incidence of bacteremia after chewing, tooth brushing and scaling in individuals with periodontal inflammation. J Clin Periodontol 2006;33:401-407.

39. Lafaurie GI, Mayorga-Fayad I, Torres MF, et al. Periodontopathic microorganisms in peripheric blood after scaling and root planing. J Clin Periodontol 2007;34: 873-879.

40. Kinane DF, Riggio MP, Walker KF, MacKenzie D, Shearer B. Bacteraemia following periodontal procedures. J Clin Periodontol 2005;32:708-713.

41. Forner L, Nielsen $\mathrm{CH}$, Bendtzen $\mathrm{K}$, Larsen $\mathrm{T}$, Holmstrup P. Increased plasma levels of IL- 6 in bacteremic periodontis patients after scaling. J Clin Periodontol 2006;33:724-729.

42. Ide M, Jagdev D, Coward PY, Crook M, Barclay GR, Wilson RF. The short-term effects of treatment of chronic periodontitis on circulating levels of endotoxin, C-reactive protein, tumor necrosis factor-alpha, and interleukin-6. J Periodontol 2004;75:420-428.

43. Krupa FG, Faltin D, Cecatti JG, Surita FG, Souza JP. Predictors of preterm birth. Int J Gynaecol Obstet 2006;94:5-11.

44. Tonetti MS, D'Aiuto F, Nibali L, et al. Treatment of periodontitis and endothelial function. N Engl J Med 2007;356:911-920.

45. Yang YM, Wang HH. Life and health concerns of Indonesian women in transnational marriages in Taiwan. J Nurs Res 2003;11:167-176.

46. Goffinet F. Primary predictors of preterm labour. BJOG 2005;112(Suppl. 1):38-47.

47. Williams CE, Davenport ES, Sterne JA, Sivapathasundaram V, Fearne JM, Curtis MA. Mechanisms of risk in preterm low-birthweight infants. Periodontol 2000 2000;23:142-150.

48. Blondel B, Dutilh P, Delour M, Uzan S. Poor antenatal care and pregnancy outcome. Eur J Obstet Gynecol Reprod Biol 1993;50:191-196.

Correspondence: Dr. Hui-Chen Chan, North University Avenue, Department of Orthodontic and Pediatric Dentistry, School of Dentistry, University of Michigan, Ann Arbor, MI 48109-1078. E-mail: pedochan@umich.edu.

Submitted September 13, 2009; accepted for publication February 25, 2010. 